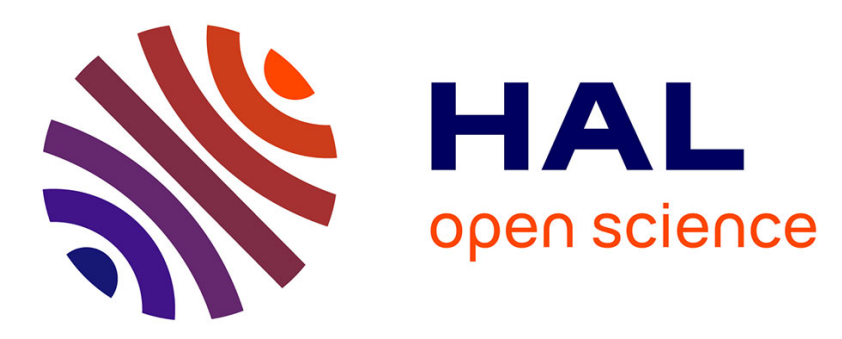

\title{
Transpired turbulent boundary layers: a general strategy for RANS turbulence models
}

François Chedevergne, Yann Marchenay

\section{To cite this version:}

François Chedevergne, Yann Marchenay. Transpired turbulent boundary layers: a general strategy for RANS turbulence models. Journal of Turbulence, 2019, 20 (11-12), pp.681-696. 10.1080/14685248.2019.1702198 . hal-02491800

\section{HAL Id: hal-02491800 \\ https://hal.science/hal-02491800}

Submitted on 26 Feb 2020

HAL is a multi-disciplinary open access archive for the deposit and dissemination of scientific research documents, whether they are published or not. The documents may come from teaching and research institutions in France or abroad, or from public or private research centers.
L'archive ouverte pluridisciplinaire HAL, est destinée au dépôt et à la diffusion de documents scientifiques de niveau recherche, publiés ou non, émanant des établissements d'enseignement et de recherche français ou étrangers, des laboratoires publics ou privés. 


\title{
Transpired turbulent boundary layers : a general strategy for RANS turbulence models
}

\author{
François Chedevergne ${ }^{\mathrm{a}}$ and Yann Marchenay ${ }^{\mathrm{a}}$
}

${ }^{a}$ ONERA, Department Multi-Physics for Energetics, 2 avenue Edouard Belin, 31055, Toulouse Cedex 4

\section{ARTICLE HISTORY}

Compiled November 28, 2019

\begin{abstract}
Transpired boundary layers are of major interest for many industrial applications. Although well described, there is no turbulence model specifically dedicated to the prediction of boundary layers for both blowing and suction configurations. Revisiting closure relations of turbulence models, a general strategy was established to recover Stevenson's law of the wall that described the behavior of transpired boundary layers in the wall region. The methodology is applied to the $k-\omega$ SST turbulence model and compared to Wilcox's correction, only applicable to blowing cases. A version of the proposed correction, more suited to RANS solvers, was also derived. Several experimental boundary layer configurations with blowing or suction were computed using both versions of the correction. The good agreements observed on all cases prove the relevance and the efficiency of the present strategy.
\end{abstract}

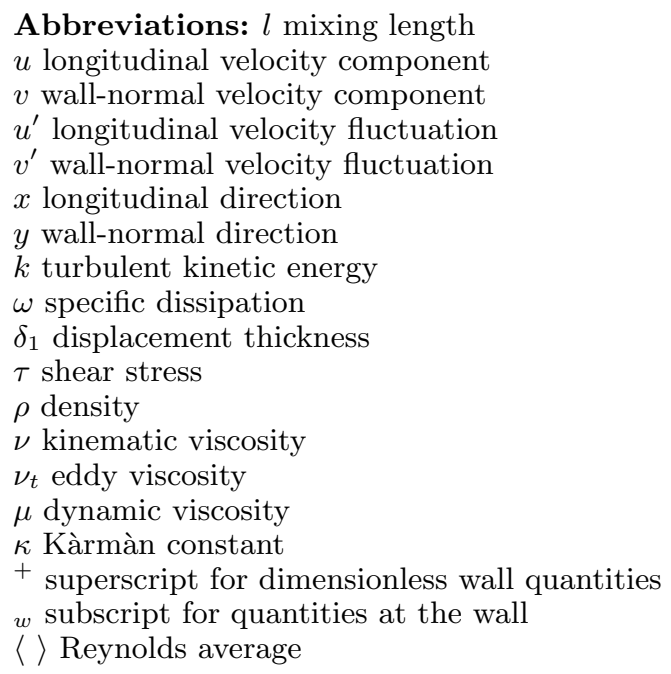

\section{KEYWORDS}

Transpired boundary layers ; blowing; suction ; turbulence models

CONTACT F. Chedevergne Email: francois.chedevergne@onera.fr 


\section{Introduction}

Boundary layers with transpiration through the solid surface have been of considerable interest in technical applications. In the present context, the word "transpiration" embrace both suction, for which the direction of the flow normal to the surface at the interface is into the surface, and at the opposite, blowing cases when the flow is directed out of the surface. Transpiration is being extensively investigated as a way to enhance the cooling of surfaces or drag reduction (blowing) or to control boundary layers separation and to delay laminar/turbulent transition (suction) in aeronautic applications.

The first significant works on the turbulent transpired boundary layer date back to the 50's $[1,2]$ and many other contributors [3-13] join the effort during the next two decades. Effects of favorable and adverse pressure gradients, roughness, fluid properties, non-uniformity, chemical species were explored in experiments leading to a rather good understanding of the mechanisms at play in turbulent boundary layers over transpired surfaces.

For bidimensionnal incompressible turbulent boundary layer developing on a flat plate with or without pressure gradients, velocity profiles behave in an opposite manner between suction and blowing cases, with respect to the sign of the wall-normal velocity. However a common formalism relying on the Prandtl mixing length hypothesis, but that can be directly deduced from a dimensionnal analysis [14], is usually employed to describe both cases. It results in a specific law of the wall, with a so-called bilogarithmic region, for these boundary layer flows which can take several forms $[1,2,15-18]$. Differences between the expressions arise from integration constants and a summary was made by Stevenson [19]. Rubesin [2] pointed out that both the mixing length parameter $\kappa$ and the integration constant should in general be regarded as functions of the suction or blowing rate. There exists other logarithmic forms, similar to that obtained without transpiration, which were deduced from asymptotic match expansions $[4,20]$, without questioning the widespread bilogarithmic form.

If considerable efforts were put to document the transpired turbulent boundary layer from experiments, far less was done on the modelling side. Using the mixing length approach, several contributions derived boundary layer closures [20-22] to account for transpiration effects on turbulence, one of the most emblematic model of that kind being the Cebecci-Smith model [23]. Concerning RANS turbulence models, except Wilcox's correction [24,25] for blowing cases, there are no specific developments to adress transpiration effects on flows. The reason for this observation is that without corrections, turbulence models behave more or less satisfactorily on boundary layers with blowing or suction when a mass flow rate is imposed as a boundary condition. To improve some specific behaviors, modifications of the injection boundary conditions mimicking the porous surface were succesfully tested by Belletre [26]. More recently, Hink [27] proposed an extension of the Wilcox's correction to the Spalart-Allmaras model [28] by exploiting an analogy with the roughness correction of Spalart and Aupoix [29]. All existing corrections for turbulence models are restricted to blowing cases and there exists neither generic correction nor strategy to account for transpiration effects in a RANS context.

After recalling the principles leading to the bilogarithmic law in section 2, the paper presents in section 3 the boundary layer model of Cebeci and Smith [23] and Wilcox's correction [24] for $k-\omega$ RANS models which are among the most widespread models accouting for transpiration effects. In section 4 the strategy to reproduce the theoretical behavior of transpired turbulent boundary layers in turbulence models is exposed. 
The strategy is then applied to the $k-\omega$ SST [30] turbulence models. Section 5 gives some validation examples.

\section{Stevenson's law of the wall}

We consider a turbulent boundary layer developing on a flat plate without pressure gradient, where $x$ denotes the longitudinal direction and $y$ the wall-normal direction. The flow is bidimensionnal, steady and incompressible. The free-stream velocity $U_{\infty}$ is therefore constant and a uniformly distributed transpiration velocity $v_{w}$ is applied at the wall. Positive values of $v_{w}$ represent blowing cases while negative values stand for suction cases. The transpiration rate $F$ is defined by $v_{w} / u_{\infty}$ since the injected fluid is assumed to be similar and at the same temperature as the mainflow, i.e. with the same density.

As evidenced by Andersen [13], Simpson [9] or Baker and Launder [12], in the inner region of boundary layers submitted to transpiration, a linear evolution of the mixing length $l$ is observed with respect to the wall distance $y$. The definition of $l$ is given by the following relation:

$$
-\left\langle u^{\prime} v^{\prime}\right\rangle=l^{2}\left|\frac{\partial u}{\partial y}\right| \frac{\partial u}{\partial y}
$$

and it is found that:

$$
l=\kappa y
$$

From this observation, a mathematical model was derived [19] for the velocity profile. Neglecting convective transport and assuming the vertical velocity component as constant in the inner region, the momentum and continuity equations may be combined to yield:

$$
\tau-\tau_{w}=\rho v_{w} u
$$

Above the viscous sublayer, the molecular contributions to the shear stress are negligible, the total stress $\tau$ reduces to the turbulent stress $\tau_{t}$ which by the mixing-length hypothesis may be replaced by:

$$
\tau_{t}=\rho l^{2}\left|\frac{\partial u}{\partial y}\right| \frac{\partial u}{\partial y}
$$

Equations (1)-(4) may be combined to give:

$$
\frac{d y^{+}}{\kappa y^{+}}=\frac{d u^{+}}{\sqrt{1+v_{w}^{+} u^{+}}}
$$

Wall quantities are turned dimensionless using the friction velocity $u_{\tau}=\sqrt{\tau_{w} / \rho}$ and the kinematic viscosity $\nu$. This equation is valid only as long as $v_{w}^{+} u^{+}>-1$, which is always satisfied for blowing configurations. Integration of (5) gives the following 
expression for the law of the wall:

$$
\frac{2}{v_{w}^{+}}\left(\sqrt{1+v_{w}^{+} u^{+}}-1\right)=\frac{1}{\kappa}\left(\ln y^{+}+C\left(v_{w}^{+}\right)\right)
$$

Eq. (6) reduces to the standard logarithmic law of the wall for $v_{w}^{+}=0$ as long as:

$$
C(0)=B
$$

This historical approach for the scaling law of turbulent transpired boundary layers was reconsidered by Vigdorovich [14] who obtained the same expression (6) using a dimensionnal analysis but without invoking any turbulent mechanisms such as the linearity of the mixing length. Moreover, $C$ was found to be depending on $v_{w}^{+}$and the asymptotic behaviors of $C$ were determined [14]. Later on, Vigdorovich [18] provided a second order development for $C$ with respect to $v_{w}^{+}$for turbulent boundary layers with suction.

\section{Cebeci-Smith model and Wilcox's correction}

The Cebecci-Smith (CS) algebraic turbulence model [23,31] has been tuned to be able to capture pressure gradient and transpiration effects on compressible boundary layer flows. Based on the Prandtl mixing length hypothesis, Cebeci and Smith used a two-layers eddy viscosity model:

$$
\nu_{t}= \begin{cases}{\left[\kappa y\left(1-e^{-y / A}\right)\right]^{2} \frac{\partial u}{\partial y}} & \text { for } y \leq y_{c} \\ 0,0168 u_{\infty} \delta_{1} & \text { for } y>y_{c}\end{cases}
$$

where $\delta_{1}$ is the displacement thickness and $y_{c}$ is the matching point insuring the continuity of $\nu_{t}$.

Correction function $A$ reads:

$$
\begin{aligned}
& A=A^{+} \frac{\nu}{u_{\tau} N} \sqrt{\frac{\rho}{\rho_{p}}} \\
& N^{2}=\frac{\nu}{\nu_{\infty}} \frac{\rho_{\infty}}{\rho} \frac{p^{+}}{v_{w}^{+}}\left(1-e^{11.8 \frac{\mu_{p}}{\mu} v_{w}^{+}}\right)+e^{11.8 \frac{\mu_{p}}{\mu} v_{w}^{+}}
\end{aligned}
$$

with the dimensionless pressure gradient $p^{+}$defined as:

$$
p^{+}=\frac{\nu_{\infty}}{u_{\tau}^{3}} u_{\infty} \frac{\mathrm{d} u_{\infty}}{\mathrm{d} x}
$$

This model was validated on various boundary layer configurations, in particular blowing and suction configurations were investigated successfully. Although the CebeciSmith model is algebraic and only applicable to boundary layers, it can serve as reference for comparisons.

Wilcox [24] developed a boundary condition applicable to $k-\omega$ two-equations turbulence models to account for blowing effects. Following conclusions drawn by Andersen 
et al. [13] from their experiments with mass injection, Wilcox relates the specific dissipation $\omega$ at the wall to the wall-normal velocity $v_{w}$. The correction consists in a modification of the boundary condition for the specific dissipation:

$$
\begin{gathered}
\omega=\frac{u_{\tau}^{2}}{\nu} S_{b} \\
S_{b}=\frac{20}{v_{w}^{+}\left(1+5 v_{w}^{+}\right)}
\end{gathered}
$$

Coefficient 20 is changed to 25 in the revised version of the model [25]. The wall normal injection $v_{w}$ lowers the specific dissipation rate and increases the eddy viscosity so that the slope of the velocity profile is altered, in a similar manner to a modification of the Kàrmàn "constant" $\kappa$ in the logarithmic law of the wall. Function $S_{b}$ was calibrated to match velocity profiles documented by Andersen et al. [13]. Wilcox's correction (11) is suitable to any $k-\omega$ turbulence model, especially the $k-\omega$ SST model of Menter [30].

At this stage, it is interesting to compute boundary layers with the $k-\omega$ SST with and without Wilcox's correction for blowing configurations to highlight self-similar behaviors in the bilogarithmic region. Several values of blowing rate $F$ are considered for illustrations and are taken equal to that of Andersen [13]. Figure 3 plots the velocity

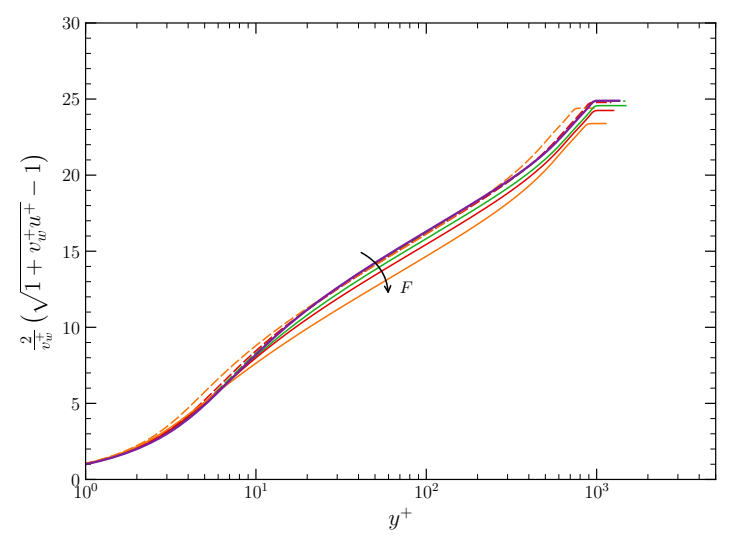

Figure 1. Velocity profiles for different blowing rates $F$ in scaling variables. Illustration of the evolution of $C$ with respect to $v_{w}^{+}$. Dashed lines (- - ) are results obtained with the uncorrected $k-\omega$ SST model and solid lines (-) with Wilcox's correction (11). Colors indicate varying blowing rates $F$ similarly to figure $2 \mathrm{~b}$.

profiles using the scaling law defined in eq. (6). All velocity profiles exhibit a linear behavior above the viscous sublayer indicating that the similarity is a universal property of the boundary layer equations with or without blowing (transpiration by extension). The linearity of the mixing length is always verified. However, if all uncorrected velocity profiles tend to collapse in a unique curve in the logarithmic region, profiles obtained with Wilcox's correction illustrate the dependency on $F$, or equivalently on $v_{w}^{+}$, of $C$ (6). This drop of $C$ as $F$ increases is directly related to the wall friction. Wilcox's correction changes the boundary condition for $\omega$ so that the turbulent contribution to the friction is modified. The uncorrected $k-\omega$ SST model is such that $C\left(v_{w}^{+}\right) \equiv B$ which leads to an underestimation, respectively overestimation, of the the friction coefficient for blowing and suction boundary layers as shown in section 5 . 


\section{Modeling strategy}

Standard RANS turbulence models are all calibrated to recover the logarithmic region of boundary layers developing over smooth surfaces without transpiration. The existence of the logarithmic region implies that the mixing lenght $l$ in the inner region is linear. As alluded earlier, experiments $[9,12,13]$ proved the relevance of the mixing length concept for the transpired boundary layer and the existence of a linear behavior in the inner region. The strategy proposed to develop a general correction for RANS turbulence models is to reconsider turbulence models calibration to account for transpiration effects. Closure relations between model's coefficients established for the inner region of the boundary layer are revisited to include additional terms directly depending on the transpiration velocity $v_{w}$.

The $k-\omega$ turbulence model family is considered. In the inner region, the momentum equation is given by eq. (3) while the turbulent kinetic energy $k$-equation still reduces to a balance between production and dissipation at the dominant order, i.e. $\beta^{*} \omega k=\nu_{t}\left(\frac{\partial u}{\partial y}\right)^{2}$. However, the $\omega$-equation must be rewritten including the convective term due to transpiration:

$$
v_{w} \frac{\partial \omega}{\partial y}=\gamma\left(\frac{\partial u}{\partial y}\right)^{2}-\beta \omega^{2}+\sigma_{\omega} \frac{\partial}{\partial y}\left(\nu_{t} \frac{\partial \omega}{\partial y}\right)
$$

where $\gamma, \beta, \beta^{*}$ and $\sigma_{\omega}$ are the closure coefficients of the $k-\omega$ model. The value $\beta^{*}=0.09$ is already fixed by the ratio $\tau / k$ in the logarithmic region [32] whereas $\beta=5 \beta^{*} / 6$ is imposed to recover homogeneous isotropic turbulence decay.

The solution consistent with this set of simplified equations is provided by Stevenson's law of the wall, namely:

$$
\frac{\partial u}{\partial y}=\frac{u_{\tau}^{*}}{\kappa y} ; \quad \nu_{t}=\kappa y u_{\tau}^{*} \quad ; \quad k=\frac{u_{\tau}^{* 2}}{\sqrt{\beta^{*}}} ; \quad \omega=\frac{u_{\tau}^{*}}{\sqrt{\beta^{*}} \kappa y}
$$

with $u_{\tau}^{*}=u_{\tau} \sqrt{1+v_{w}^{+} u^{+}}$a function of $y^{+}$.

A unique relation exists between coefficients while introducing solution (13) into eq. (12). The following relation must hold:

$$
\gamma=\frac{\beta}{\beta^{*}}-\frac{\sigma_{\omega} \kappa^{2}}{\sqrt{\beta^{*}}}\left[1-\frac{3}{2 \kappa} \frac{v_{w}}{u_{\tau}^{*}}+\frac{1}{4 \kappa^{2}}\left(\frac{v_{w}}{u_{\tau}^{*}}\right)^{2}\right]-\frac{\kappa}{\sqrt{\beta^{*}}} \frac{v_{w}}{u_{\tau}^{*}}+\frac{1}{2 \sqrt{\beta^{*}}}\left(\frac{v_{w}}{u_{\tau}^{*}}\right)^{2}
$$

Obviously, the original relation for the non-transpired case is recovered for $v_{w}=0$. At this stage, two options can be considered since coefficients $\gamma$ and $\sigma_{\omega}$ remain unfixed. First, the whole correction can be supported by coefficient $\gamma$ while $\sigma_{\omega}$ will be choosen according to arguments already invoked for non-transpired boundary layers. Wilcox [24] reported that the wake region of a non-transpired boundary layer is directly influenced by the value of $\sigma_{\omega}$. The second option is to distribute the correction terms between $\gamma$ and $\sigma_{\omega}$. The idea is to maintain the dissipation term of eq. (12) unchanged for non-transpired configuration in order to keep the correct behavior in the wake region. Numerical experimentation proved that the second option allows better agreement with measurements in the outer region of the computed boundary layers. 
Finally, the proposed correction reads:

$$
\gamma=\gamma_{0}-\frac{\kappa}{\sqrt{\beta^{*}}} \frac{v_{w}}{u_{\tau}^{*}}+\frac{1}{2 \sqrt{\beta^{*}}}\left(\frac{v_{w}}{u_{\tau}^{*}}\right)^{2}=\frac{\sigma_{\omega_{0}}}{1-\frac{3}{2 \kappa} \frac{v_{w}}{u_{\tau}^{*}}+\frac{1}{4 \kappa^{2}}\left(\frac{v_{w}}{u_{\tau}^{*}}\right)^{2}}
$$

where $\gamma_{0}=\frac{\beta}{\beta^{*}}-\frac{\sigma_{\omega_{0}} \kappa^{2}}{\sqrt{\beta^{*}}}$ and $\sigma_{\omega_{0}}$ are the original values of $\gamma$ and $\sigma_{\omega}$ calibrated on non-transpired boundary layers.

The correction given by expressions (15) operates differently between blowing and suctions cases. For blowing configurations, imposing a positive value $v_{w}^{+}$at the wall implies an asymptotic behavior for the turbulent kinetic energy so that $k^{+} \rightarrow+\infty$ for $v_{w}^{+} \rightarrow+\infty$ since $u_{\tau} \rightarrow 0$. In parallel, the dissipation $\varepsilon^{+}$also tends to infinity. For a given $v_{w}^{+}$value, the proposed correction reduces the peak values of $k^{+}$and $\varepsilon^{+}$but the distribution of the ratio $\frac{k^{+^{2}}}{\varepsilon^{+}}$across the boundary layer is increased. Thus, the correction increases $\nu_{t}^{+}$and finally limits the effect of introducing a wall normal velocity component $v_{w}^{+}$on the wall friction. For suction configurations, $k^{+}$and $\varepsilon^{+}$tend to zero when $v_{w}^{+} \rightarrow-\infty$. When $v_{w}^{+}$is fixed, the correction in eq. (15) still lowers $k^{+}$and $\varepsilon^{+}$profiles but $k^{+^{2}}$ decreases faster than $\varepsilon^{+}$. The resulting eddy viscosity profile $\nu_{t}^{+}$is lowered just like the friction level compared to the uncorrected case. As a consequence, the resulting velocity profiles obtained with corrections (15) conform to the similarity profile of Stenvenson's law but with variable $C$ values depending on $v_{w}^{+}$contrary to the uncorrected profiles of figure 3 .

Relation (15) can hardly be used in a straightforward manner in a Navier-Stokes solver since $u_{\tau}^{*}$ depends on $y$ and $u_{\tau}$. To circumvent this problem, rather strong assumptions are made to relate the ratio $v_{w} / u_{\tau}^{*}$ to the transpiration rate $F$ :

$$
\frac{v_{w}}{u_{\tau}^{*}} \approx \delta_{v} \sqrt{\frac{\left|v_{w}^{+}\right|}{u^{+}}} \approx \delta_{v} \sqrt{\frac{\left|v_{w}\right|}{u_{\infty}}}=\delta_{v} \sqrt{|F|}
$$

where $\delta_{v}$ is the sign of $v_{w}$. The asymptotic behavior for blowing as $y \rightarrow+\infty$ of the simplified model (13) gives $v_{w}^{+} u^{+} \gg 1$ and leads to:

$$
\frac{v_{w}}{u_{\tau}^{*}}=\frac{v_{w}^{+}}{\sqrt{1+v_{w}^{+} u^{+}}} \simeq \sqrt{\frac{v_{w}^{+}}{u^{+}}}
$$

The second approximation may be seen as a result of the matching between the inner region and the outer region of the boundary layer where the velocity is of the order of $u_{\infty}$. Hence eq. (17) yields:

$$
\sqrt{\frac{v_{w}^{+}}{u^{+}}} \simeq \sqrt{F}
$$


Nevertheless, when plotting $v_{w} / u_{\tau}^{*}$ with respect to $y^{+}$for several blowing and suction cases, $\delta_{v} \sqrt{|F|}$ does not always appear to be a reasonable representative mean value. Consequently, replacing $\frac{v_{w}}{u_{\tau}^{*}}$ by $\delta_{v} \sqrt{|F|}$ provides good results on blowing cases but induces substantial degradation for boundary layers with suctions as the first approximation is no longer valid. The reason lies in the modification of $\sigma_{\omega}$ in eq. (15). For boundary layers with blowing, the logarithmic region is mainly dominated by inertial effects while for suction cases the dissipation plays a more important role. The approximation made in eq. (16) considerably affects $\sigma_{\omega}$ so that it is preferable to drop this part of the correction. Additionally, from experimental profiles detailed in section 5 , a reasonable overall approximation for $v_{w} / u_{\tau}^{*}$ is found to be:

$$
\frac{v_{w}}{u_{\tau}^{*}} \approx\left(\delta_{v}-0,3\right) \sqrt{|F|}
$$

The correction of eq. (15) and its complementary version using eq. (19) are applied to the $k-\omega$ SST turbulence model. This model includes two sets of coefficients blended by function $F_{1}[30]$. As the correction is devoted to the behavior of flows in the wall region, only the first set of coefficient for which $F_{1}=1$ must be altered. Note that the baseline version (BSL) of the model [30] does not produce identical results on blowing configurations. The shear stress limiter of the SST version is activated in the inertial region of the boundary layer profile. The positive wall-normal velocity component $v$ contributes to the increase of the turbulent shear $-\overline{u^{\prime} v^{\prime}}$ and the ratio $-\overline{u^{\prime} v^{\prime}} / k$ tends to exceed $a_{1}=0.31$.

Typical values for $|F|$ do not exceed 0.01 , otherwise relaminarization or separation occur. Since $\gamma_{0}=0.553$ and $\sigma_{\omega_{0}}=0.5$ for the inner part of the $k-\omega$ SST model, the correction stands for a modification of less than $22 \%$ for $\gamma_{0}$ and less than $54 \%$ for $\sigma_{\omega_{0}}$.

\section{Validation}

Model validation was performed using ONERA's two-dimensional boundary layer code CLICET [33]. An automatic grid adaptation procedure insures grid converged results. Some transient can be observed at the beginning of the computations due to the initial boundary layer profile generation from a locally self-similar solution. The code was extensively used by Aupoix for many boundary layer applications such as roughness effects modelling [29,34-36].

The objective of the validation section is twofold. First, the developed correction (15) must be assessed against existing models, i.e. the Cebeci-Smith model and the $k-\omega$ SST coupled to the Wilcox's correction, and also against experiments. The CS model can be seen as a reference model for transpired boundary layers as it relies directly on the mixing length concept that drives the desired behavior of Stevenson's law of the wall. For this reason, experimental results will always be presented together with results obtained with the CS model on figures.

A set of experimental data, usable for transpired boundary layer applications, was identified. The very complete collection of Stanford University obtained in the 60's and 70's by Kays, Moffat and co-workers constitutes a recognized reference. Among the variety of boundary layer configurations tested during that period, Andersen's work [13] provides accurate measurements for blowing cases without pressure gradients. These data have already served to validate models such as Wilcox's correction (11) and form 
the first validation test case in section 5.1. For zero pressure gradient boundary layers with suction, the work by Ferro $[37,38]$ was retained and is used for comparisons in section 5.2. To complete the validation cases, the effect of an adverse pressure gradient on the transpired turbulent boundary layer is examined. Andersen [13] also collects data on blowing and suction cases with adverse pressure gradients. Theses configurations are investigated in section 5.3.

\subsection{Blowing without pressure gradient}

Andersen [20] studied turbulent boundary layers developing on a flat plate without pressure gradient but with the presence of mass injection. Four blowing rates $F>0$ were investigated but only three are examined here, although the whole dataset was used by Wilcox [24] to develop his correction. For the largest value $F=0.008$, the friction coefficient $C_{f}$ is very low and hard to estimate experimentally. Incidentally, data are not presented in Andersen's article [13]. For each of the blowing rate, friction coefficients were obtained at several longitudinal positions $x$. Profiles at $x=90$ inch., i.e. $R e_{x} \approx 1.4 \times 10^{6}$, were also measured.

Computations with and without transpiration corrections were performed on all the configurations. For these computations the blowing rate is applied as a boundary condition through a constant wall-normal injection velocity. $k-\omega$ SST computations were performed using the Wilcox's correction, the present correction with eq. (15) and finally using eq. (19). As expected, results obtained with the CS model are in good agreement with the measurements.

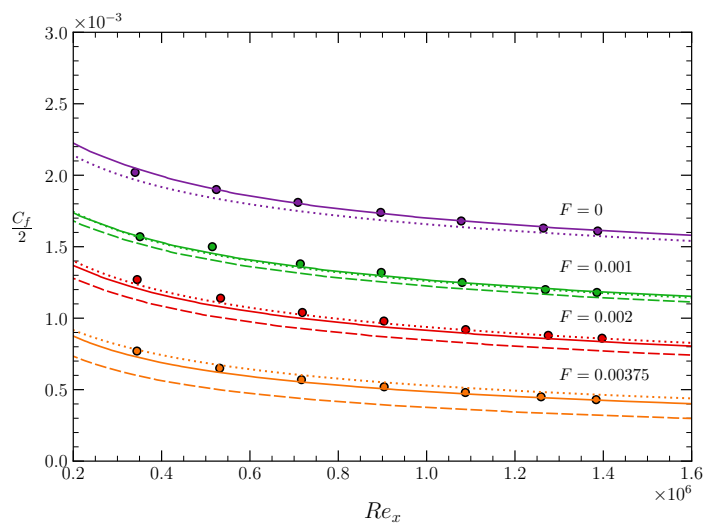

(a) friction coefficient

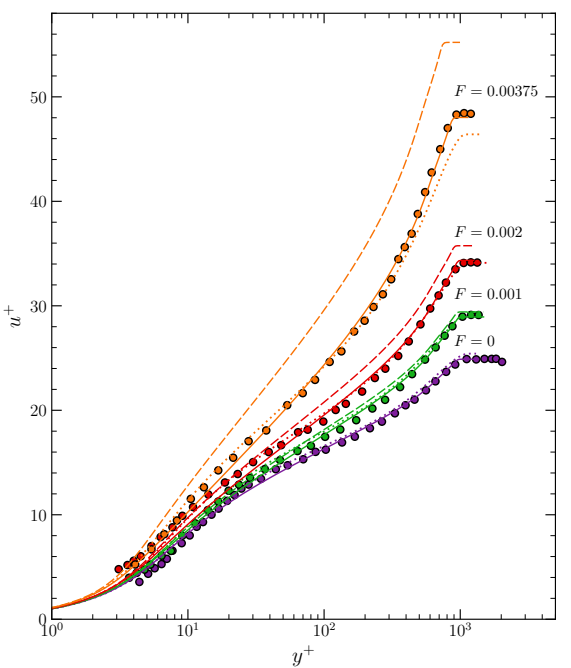

(b) velocity profile

Figure 2. Zero pressure gradient boundary layers for several blowing rate $F$. Symbols represent experimental data of Andersen [13]. Dotted lines (.......) are results obtained with the CS model, dashed lines (- - -) with the uncorrected $k-\omega$ SST model and solid lines (-) with Wilcox's correction (11)

Figures $2 \mathrm{a}$ and $2 \mathrm{~b}$ show the efficiency of Wilcox's correction on $k-\omega$ SST computations. Without correction, the injection condition tends to lower the friction coefficient compared to measurements which moves the velocity profiles upward since $u_{\infty}^{+}=\sqrt{2 / C_{f}}$. 


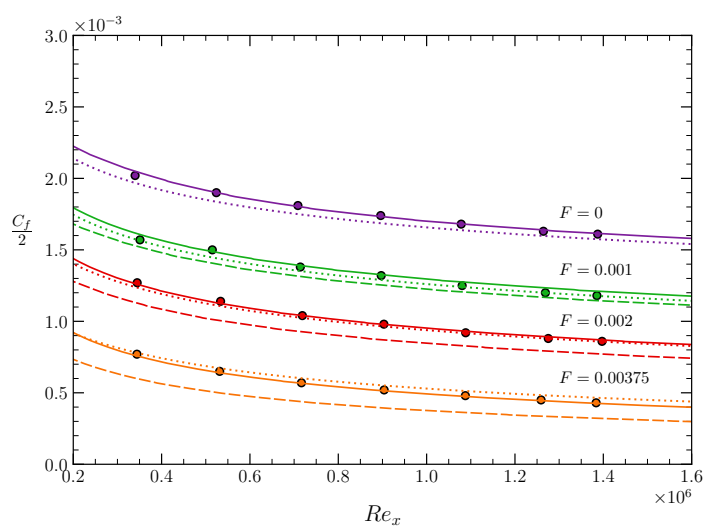

(a) friction coefficient

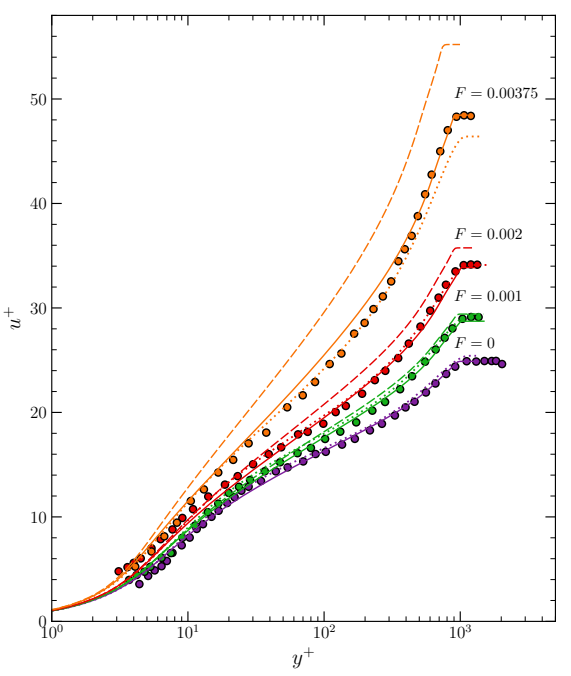

(b) velocity profile

Figure 3. Zero pressure gradient boundary layers for several blowing rate $F$. Symbols represent experimental data of Andersen [13]. Dotted lines (.......) are results obtained with the CS model, dashed lines (- - -) with the uncorrected $k-\omega$ SST model and solid lines (-) with correction of eq. (15)

Similar results are obtained using the proposed modification of the model coefficients (15). For $F=0.00375$, the modification of the slope in the bi-logarithmic region imposed by the new values of the coefficients is clearly visible. Remark, that the new correction does not act similarly to that of Wilcox. Even if both corrected profiles fairly reproduce the measured ones, the profile shapes are different revealing the different nature of the mechanisms at play in the two corrections. Wilcox's correction acts on the eddy viscosity from the wall while eq. (15) imposes the distribution of $\nu_{t}$ along the profile.

The introduction of approximation (19) does not alter the overall agreement as shown on figure $4 \mathrm{a}$ and $4 \mathrm{~b}$. A slight degradation is visible for $F=0.001$ suggesting that the approximation better works for high values of $F$. Even though Wilcox's correction produces best results as it has been calibrated on these experiments, the strategy adopted to develop the present correction seems effective.

\subsection{Suction without pressure gradient}

More interestingly, the strategy exposed in this paper is now applied to suction cases. Indeed, Wilcox's correction does not work for negative values of $v_{w}$ and there was no other correction to deal with suction in $k-\omega$ SST model. A recent work of Lehmkuhl et al. [39] proposes an adaption of standard law of the wall to take mass transfert effects (suction) into account but remains limited to wall function strategy. Among configurations studied by Ferro [37], we considered boundary layers developing on a flat plate without pressure gradient. The flat plate is $6.6 \mathrm{~m}$ long and the suction starts after an elongated impermeable leading edge. High Reynolds numbers are reached at the end of this long plate. Without suction the Reynolds number based on the momentum thickness $\theta$ at the end of the plate can be up to more that $R e_{\theta}=21000$ in Ferro's 


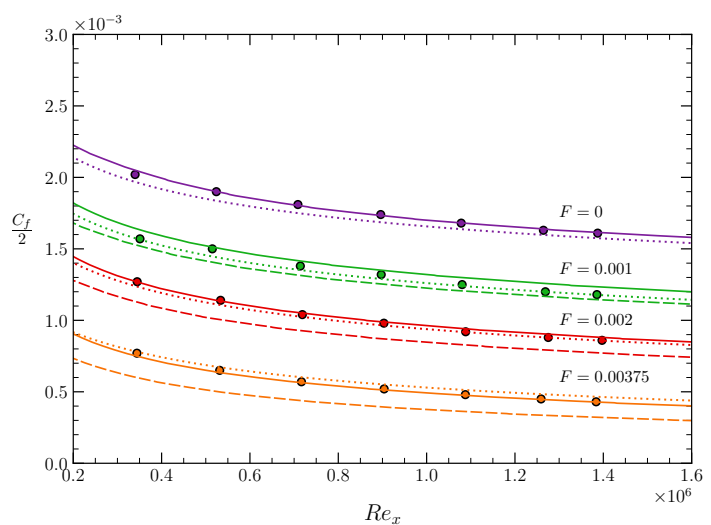

(a) friction coefficient

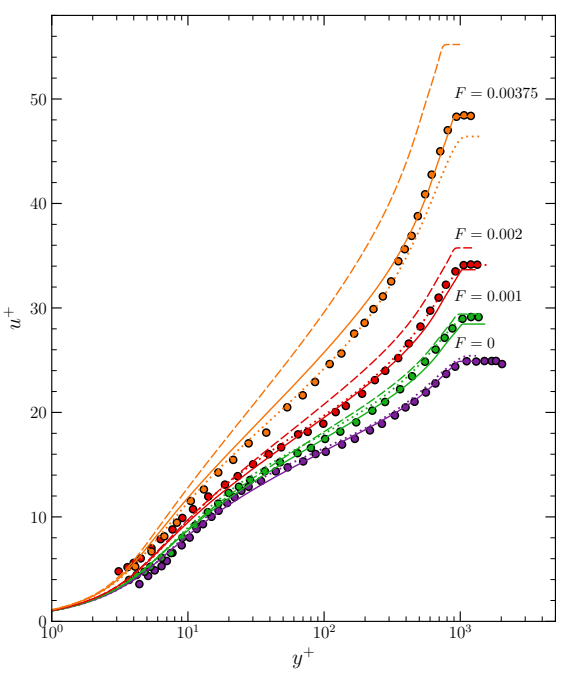

(b) velocity profile

Figure 4. Zero pressure gradient boundary layers for several blowing rate $F$. Symbols represent experimental data of Andersen [13]. Dotted lines (.......) are results obtained with the CS model, dashed lines (- - -) with the uncorrected $k-\omega$ SST model and solid lines (-) with correction of eq. (15) and using eq. 19)

Table 1. Friction coefficient $C_{f}\left(\times 10^{3}\right)$ in Ferro's experiments

\begin{tabular}{lcc}
\hline & $F=-0.00258$ & $F=-0.00327$ \\
\hline Exp. & 5.19 & 6.51 \\
No corr. & 5.63 & 6.87 \\
corr. (15) & 5.30 & 6.63 \\
corr. with (19) & 5.30 & 6.62 \\
\hline
\end{tabular}

experiments. Thus, the asymptotic behavior of boundary layers is achieved with or without suction. The two most intense suction cases were retained in these experiments for comparisons. The corresponding suction rate are $F=-0.00258$ and $F=-0.00328$. Profiles are extracted at the longitudinal position $x=4.8 \mathrm{~m}$. Corresponding Reynolds numbers are $R_{\theta}=5620$ and $R_{\theta}=1800$.

Ferro [37] indicates that his measurements do not match Stevenson's law of the wall. When $u_{p}^{+}=\frac{2}{v_{w}^{+}}\left(\sqrt{1+v_{w}^{+} u^{+}}-1\right)$ is plotted in a semi-logarithmic diagram, a linear region is clearly visible but the intercept differs from $B(6)$. What Ferro's experiments show is that eq. (7) is not valid for these suction cases. But, for the proposed correction, only the slope $\frac{\partial u^{+}}{\partial y^{+}}$matters, constant $B$ does not interfere. Results plotted on figures $5 \mathrm{a}$ and $5 \mathrm{~b}$ prove that the construction of the correction is correct. The experimental velocity profiles are remarkably recovered using the present correction in both forms, even better than using the CS model. On the contrary, uncorrected profiles largely depart from the measurements. In term of friction coefficient, given in table 1 , differences rise up to $8.5 \%$ without correction and reduces to $2 \%$ using relation (15). 


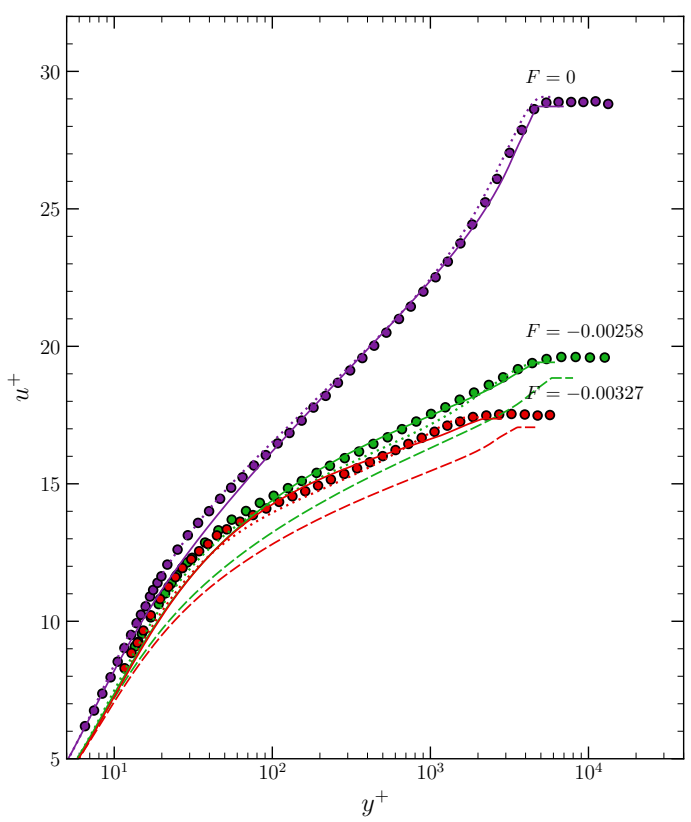

(a) correction of eq. (15)

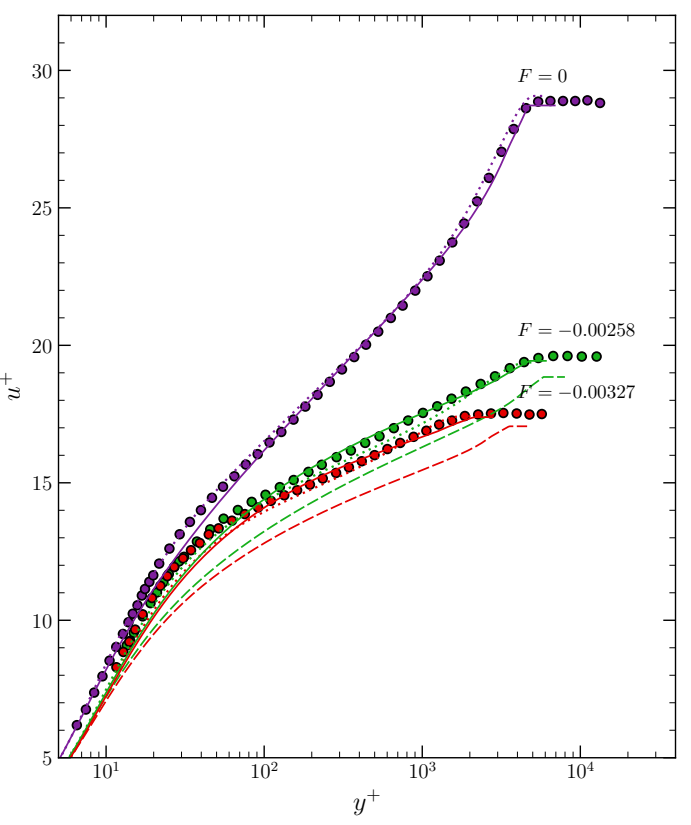

(b) correction using eq. (19)

Figure 5. Velocity profiles for zero pressure gradient boundary layers with suction. Symbols depict exprimental data obtained by Ferro [37]. Dotted lines (.......) are results obtained with the CS model, dashed lines (- - ) with the uncorrected $k-\omega$ SST model and solid lines (-) with correction

\subsection{Transpiration with adverse pressure gradient}

Effects of adverse pressure gradients on transpired boundary layers were explored by Andersen et al. $[13,20]$. Several configurations were tested where either the injection velocity or transpiration rate is constant. For the sake of simplicity, cases where $F$ is constant are retained. The imposed pressure gradient is such that the freestream velocity is given by $u_{\infty}=29.2\left(\frac{x+3}{7}\right)^{m}$ with $m=-0.15$. In addition to the nominal configuration $F=0$, three values of $|F|$ were investigated, providing six configurations with $F= \pm 0.001, F= \pm 0.002$ and $F= \pm 0.004$. The blowing case with $F=0.004$, never published but given in [20], induces very low friction coefficients with a high degree of uncertainty and is thus hardly exploitable. The five remaining cases were all simulated and results are reported in figures $6 \mathrm{a}$ to $7 \mathrm{~b}$.

A good overall agreement is observed with experiments, regardless the nature of the correction. Wilcox's correction also behaves very well on blowing configurations. However, for suction cases, computed profiles exhibit lower boundary layers thicknesses compared to measurements. There is no direct explanation from the computational side for that, whereas there were not such differences on configurations without pressure gradient in section 5.2. Additionally, it is also observed on figure 6a that for blowing configurations, especially, the last measuring points reveal a singular behavior for the friction coefficients. Instead of a plateau, a sudden slight drop appears for $R e_{x}>8 e^{5}$. The imposed pressure gradient in the computation does not permit to recover this trend. There is presumably something in the experiments that is not accounted for in the computations. For this reason, profiles are extracted at station $x=75$ inch. corresponding to $R e_{x} \approx 7 \times 10^{5}$. 


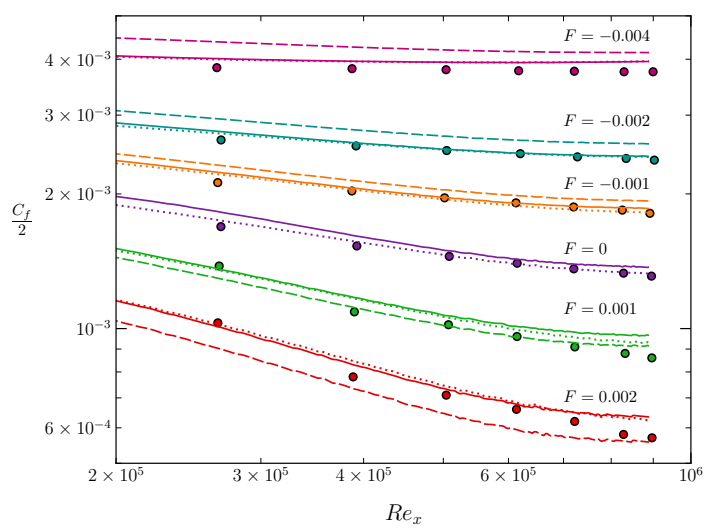

(a) friction coefficient

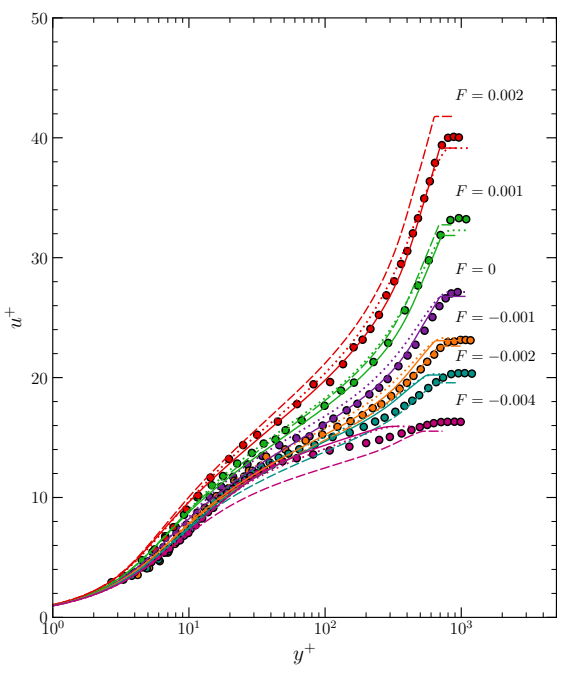

(b) velocity profiles

Figure 6. Friction coefficient and velocity profiles for blowing and suction configurations with the presence of an adverse pressure gradient. Symbols represent measurements obtained by Andersen [13]. Dotted lines ( - ) are results obtained with the CS model, dashed lines (- - ) with the uncorrected $k-\omega$ SST model and solid lines (-) with correction of eq. (15)

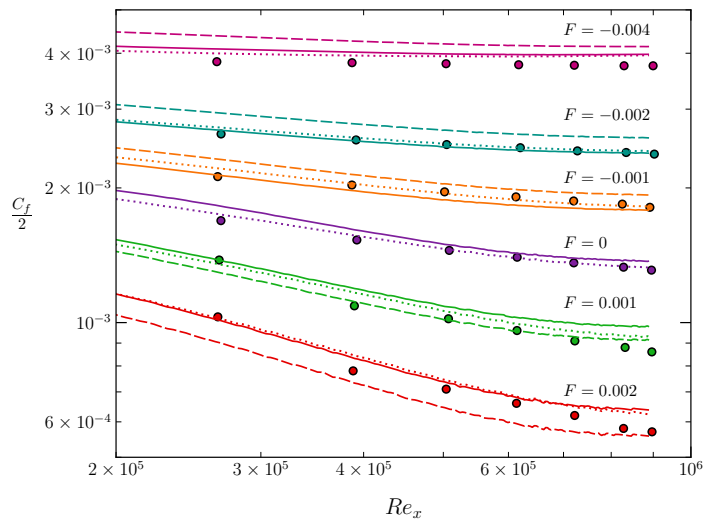

(a) friction coefficient

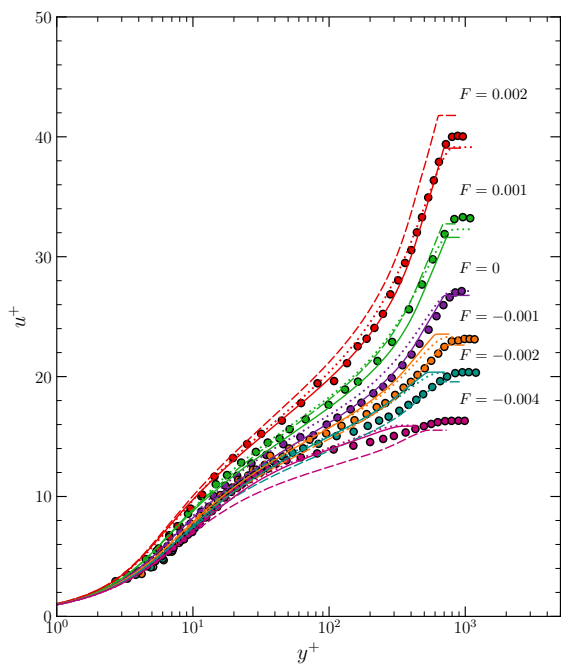

(b) velocity profiles

Figure 7. Friction coefficient and velocity profiles for blowing and suction configurations with the presence of an adverse pressure gradient. Symbols represent measurements obtained by Andersen [13]. Dotted lines ( -$)$ are results obtained with the CS model, dashed lines (- - ) with the uncorrected $k-\omega$ SST model and solid lines (-) with correction of eq. (15) and using eq. (19)

It seems that all the velocity profiles are mainly driven by the existence of a pressure gradient. Compared to previous cases without pressure gradient where the uncorrected $k-\omega$ SST model provided bad results, the present computations show a satisfactory agreement between the standard $k-\omega$ SST model and the experiments. The addition of the correction slightly modifies the velocity profiles and more significantly the $C_{f}$ 
distributions. The two forms of the correction behave as expected and improve the results in terms of friction levels. The results are very similar to those provided by the CS model, reassuring the use of the present strategy to develop a correction for transpiration effects.

\section{Conclusions}

A general strategy was proposed to account for transpiration effects on boundary layer flows by revisiting the closure relations of turbulence models. Similarly to boundary layers developing on impermeable walls for which the standard logarithmic behavior is forced by relating turbulence models coefficients to each other, the main idea is to impose a bilogarithmic behavior in the wall region given by Stevenson's law of the wall. The strategy is applied to the $k-\omega$ SST model and compared to Wilcox's correction. The latter already produces very good results on blowing cases but can not be applied to boundary layers with suction. In addition, Wilcox's correction induces a modification of $\omega$ boundary condition at the wall which is not compatible with other corrections necessitating modifications of boundary conditions such as roughness corrections [35]. The present approach removes this constraint and provides a correction that works for both suction and blowing cases.

The original correction given by eq. (15) was rewritten to make it more suitable to RANS solvers by replacing function $v_{w}^{+} u \tau / u_{\tau}^{*}$ by an expression (19) only depending on the transpiration rate $F$. In addition, results for non-transpired boundary layers are not affected by this strategy since for $v_{w}=0$ the correction is zero and the recourse to function $F_{1}$ of the $k-\omega$ SST model limits the extend of the correction to the wall region and preserves wall-free flows. Both versions of the correction were successfully tested on blowing and suction cases, with and without adverse pressure gradient.

\section{References}

[1] Mickley HS, Davis RS. Momentum transfer for flow over a flat plate with blowing. NASA; 1957. TN-4017.

[2] Rubesin MW. An analytical estimation of the effect of transpiration cooling on the heattransfer and skin-friction characteristics of a compressible, turbulent boundary layer. NASA; 1974 . TN-3341.

[3] Stevenson TN. Turbulent boundary layers with transpiration. AIAA Journal. 1964 aug; 2(8):1500-1502. Available from: https://doi.org/10.2514/3.2595.

[4] Tennekes H. Similarity laws for turbulent boundary layers with suction or injection. Journal of Fluid Mechanics. 1965 apr;21(04):689. Available from: https://doi.org/10.1017/s0022112065000423.

[5] Moffat RJ, Kays WM. The turbulent boundary layer on a porous plate: Experimental heat transfer with uniform blowing and suction. International Journal of Heat and Mass Transfer. 1968 oct;11(10):1547-1566. Available from: https://doi.org/10.1016/00179310(68)90116-6.

[6] Bradshaw P. Mixing-length velocity profile in boundary layers with transpiration. AIAA Journal. 1967 sep;5(9):1674-1675. Available from: https://doi.org/10.2514/3.4267.

[7] Jeromin LOF. A transformation for compressible turbulent boundary layers with air injection. Journal of Fluid Mechanics. 1968 jan;31(01):65. Available from: https://doi.org/10.1017/s0022112068000042.

[8] Squire LC. A law of the wall for compressible turbulent boundary layers with 
air injection. Journal of Fluid Mechanics. 1969 jul;37(03):449. Available from: https://doi.org/10.1017/s0022112069000656.

[9] Simpson RL. Characteristics of turbulent boundary layers at low reynolds numbers with and without transpiration. Journal of Fluid Mechanics. 1970 jul;42(04):769. Available from: https://doi.org/10.1017/s002211207000160x.

[10] Julien HL, Kays WM, Moffat RJ. Experimental hydrodynamics of the accelerated turbulent boundary layer with and without mass injection. Journal of Heat Transfer. 1971; 93(4):373. Available from: https://doi.org/10.1115/1.3449833.

[11] Kays WM. Heat transfer to the transpired turbulent boundary layer. International Journal of Heat and Mass Transfer. 1972 may;15(5):1023-1044. Available from: https://doi.org/10.1016/0017-9310(72)90237-2.

[12] Baker RJ, Launder BE. The turbulent boundary layer with foreign gas injection-i. measurements in zero pressure gradient. International Journal of Heat and Mass Transfer. 1974 feb;17(2):275-291. Available from: https://doi.org/10.1016/0017-9310(74)90089-1.

[13] Andersen PS, Kays WM, Moffat RJ. Experimental results for the transpired turbulent boundary layer in an adverse pressure gradient. Journal of Fluid Mechanics. 1975 may; 69(02):353. Available from: https://doi.org/10.1017/s0022112075001474.

[14] Vigdorovich I. Velocity, temperature, and reynolds-stress scaling in the wall region of turbulent boundary layer on a permeable surface. Journal of Experimental and Theoretical Physics. 2004 Nov;99(5):1028-1038. Available from: https://doi.org/10.1134/1.1842884.

[15] Black T, Sarnecki A, Britain) ARCG. The turbulent boundary layer with suction or injection. H.M. Stationery Office; 1965. Aeronautical research Council; reports and memoranda; Available from: https://books.google.fr/books?id=bvyicQAACAAJ.

[16] Stevenson TN. Inner region of transpired turbulent boundary layers. AIAA Journal. 1968 mar;6(3):553-554. Available from: https://doi.org/10.2514/3.4539.

[17] Simpson R, Whitten D, Moffat R. An experimental study of the turbulent prandtl number of air with injection and suction. International Journal of Heat and Mass Transfer. 1970 jan;13(1):125-143. Available from: https://doi.org/10.1016/0017-9310(70)90030-x.

[18] Vigdorovich I. A law of the wall for turbulent boundary layers with suction: Stevenson's formula revisited. Physics of Fluids. 2016 aug;28(8):085102. Available from: https://doi.org/10.1063/1.4960182.

[19] Stevenson TN. A law of the wall for turbulent boundary layers with suction or injection. Cranfield College of Aeronautics; 1963. 166.

[20] Andersen PS, Kays WM, Moffat RJ. The turbulent boundary layer on a porous plate: An experimental study of the fluid mechanics for adverse free stream pressure gradients. NASA; 1972. HMT-15.

[21] Kays WM. Heat transfer to the transpired turbulent boundary layer; 1971. HMT-14.

[22] Baker RJ, Launder BE. The turbulent boundary layer with foreign gas injection-II. predictions and measurements in severe streamwise pressure gradients. International Journal of Heat and Mass Transfer. 1974 feb;17(2):293-306. Available from: https://doi.org/10.1016/0017-9310(74)90090-8.

[23] Cebeci T, Smith A. Analysis of turbulent boundary layers. (Applied Mathematics and Mechanics; Vol. 15). Academic Press; 1974.

[24] Wilcox D. Reassessment of the scale-determining equation for advanced turbulence models. AIAA Journal. 1988 November;26(11):1299-1310.

[25] Wilcox D. Formulation of the $k-\omega$ turbulence model revisited. AIAA Journal. 2008 November;46(11):2823-2838.

[26] Bellettre J, Bataille F, Lallemand A. A new approach for the study of turbulent boundarylayers with blowing. International Journal of Heat and Mass Transfer. 1999 aug; 42(15):2905-2920. Available from: https://doi.org/10.1016/s0017-9310(98)00341-x.

[27] Hink R, Hannemann V, Eggers T. Extension of the spalart-allmaras one-equation turbulence model for effusion cooling problems. In: Deutscher Luft- und Raumfahrtkongress 2013; September; 2013. Available from: https://elib.dlr.de/84638/.

[28] Spalart P, Allmaras S. A one-equation turbulence model for aerodynamic flows. La 
Recherche Aérospatiale. 1994;1:5-21.

[29] Aupoix B, Spalart P. Extensions of the Spalart-Allmaras turbulence model to account for wall roughness. International Journal of Heat and Fluid Flows. 2003;24:454-462.

[30] Menter F. Two-equation eddy-viscosity turbulence models for engineering applications. AIAA Journal. 1994 August;32(8):1598-1605.

[31] Cebeci T, Smith A, Mosinskis G. Calculation of compressible adiabatic turbulent boundary layers. AIAA Journal. 1970 November;8(11):1974-1982.

[32] Bradshaw P, Ferriss D, Atwell N. Calculation of boundary-layer development using the turbulent energy equation. Journal of Fluid Mechanics. 1967;28(3):593-616.

[33] Aupoix B. CLICET - version 2015 descriptif et mode d'emploi. ONERA; 2015. Rapport Technique $1 / 24088$ DMAE.

[34] Aupoix B. A general strategy to extend turbulence models to rough surfaces - Application to Smith's $k-l$ model. Journal of Fluid Engineering. 2007 October;129(10):1245-1254.

[35] Aupoix B. Roughness Corrections for the $k-\omega$ Shear Stress Transport Model: Status and Proposals. Journal of Fluids Engineering. 2015 February;137:021202.

[36] Aupoix B. Improved heat transfer predictions on rough surfaces. International Journal of Heat and Fluid Flows. 2015 December;56:160-171.

[37] Ferro M. Experimental study on turbulent boundary-layer flows with wall transpiration [dissertation]. Stockholm: Royal Institute of Technology; 2017.

[38] Ferro M, Bengt F, Fransson J. On the scaling of turbulent asymptotic suction boundary layers. In: $10^{\text {th }}$ International Symposium on Turbulence and Shear Flow Phenomena, TSFP17; july; 2017.

[39] Lehmkuhl J, Kelm S, Bucci M, et al. Improvement of wall condensation modeling with suction wall functions for containment application. Nuclear engineering and design. 2016; 299:105-111. 